\title{
MJN THE ROLE OF WARD MANAGERS WHEN DEVELOPING CONSISTENT EVIDENCE-BASED PRACTICES IN LONG-TERM CARE FACILITY: A QUALITATIVE STUDY
}

\author{
Teija Korhonen ${ }^{1 *}$, Arja Holopainen², Anne-Maria Kanerva ${ }^{3}$, Seija Petman ${ }^{4}$, Elina Haavisto ${ }^{5}$ \\ ${ }^{1}$ Senior Lecturer, Savonia University of Applied Sciences, Kuopio, Finland \\ ${ }^{2}$ Research Director, Finnish Nursing Research Foundation, Finland \\ ${ }^{3}$ Principal lecturer, Satakunta University of Applied Sciences, Pori, Finland \\ ${ }^{4}$ Master of Health Care, Outreach youth worker, City of Rauma, Finland \\ ${ }^{5}$ Department of Nursing Science, University of Turku, Turku, Finland \\ *Corresponding Author's Email: tija.korhonen@savonia.fi
}

\begin{abstract}
Background: A ward manager performs important tasks in the implementation of consistent evidence-based practice (EBP). However, little is known about these tasks, especially in long-term care facilities. Aim: This study aimed to describe the tasks of ward managers in improving consistent EBP according to the four-phase Operational Model for Evidence-Based Practice (OMEBP). Method: Data were collected through focus group interviews (9) in each of the four phases of OMEBP. The interviews were separately conducted in two groups: (1) ward managers $(n=4)$ and $(2)$ wound contact persons (registered and practical nurses, $n=8)$; participants from the four units were present in the same interview. In addition, a third focus group [practical nurses $(\mathrm{n}=8)$ was involved in the first phase of the interview regarding the evaluation of the development needs of the current practice and their realization. The groups were interviewed after the completion of each phase of OMEBP. Data were analyzed by deductive content analysis based on the four phases of OMEBP. Results: Results described the tasks of ward managers in the four-phase OMEBP. These tasks included informing, mentoring, taking responsibility, supporting the nursing staff, reminding, and consulting. Conclusions: Ward managers perform several tasks during the implementation of consistent EBP. Management of EBP requires several knowledge and skills.
\end{abstract}

Keywords: Ward Manager, Evidence-Based Practice, Consistent Practice, Operational Model for EvidenceBased Practice (OMEBP)

\section{INTRODUCTION}

Globally, there is a growing interest in improving the use of healthcare policy that targets funding toward programs and practices, which are based on the best available evidence (Supplee \& Metz, 2015; Langlois, Daniels \& Akl, 2018). Evidence is needed in assessing the effects of interventions that aim to reduce health inequities across populations (Langlois, Daniels \& Akl, 2018) and in ensuring the quality of care. Such evidence is useless if not incorporated in decision-making in healthcare (Andermann et al., 2016). For example, the Institute of Medicine (2009) has set the goal wherein by $2020,90 \%$ of clinical decisions in healthcare practices must be supported by timely, accurate, and up-to-date clinical information.

The excuse that patient care varies depending on who is caring for the patients or where the patients are being cared for is unacceptable. This variation in care can result in poor patient outcomes because of interventions that can be harmful, ineffective, or useless to the patients' health (Kalisch et al., 2011; Stevens, 2013). Therefore, improving consistent evidence-based practices (EBPs) is crucial in ensuring that decisionmaking is based on the best possible evidence to eliminate unjustified variation in healthcare practices (Heiwe et al., 2011). Consistent EBP is usually based on 
systematic reviews or on evidence-based clinical guidelines and is the basis in developing concrete instructions, such as checklists, protocols, and care protocols (Innis et al., 2015; Veermah, 2016; Jylhä et al., 2017). These tools are helpful in disseminating EBP to clinical settings and in decision-making (Jun, Kovner \& Simpfel, 2016).

Different models have been developed for evidence dissemination and implementation (van Achterberg, Schoonhoven \& Grol, 2008; Wang, Kao \& Lin, 2015; Stevens 2013). In the present study, the Operational Model for Evidence-Based Practice (OMEBP) was used as a framework for the improvement in consistent practice. This model consists of four phases: (1) determination of the development needs of the current practice to assess whether it is in line with the best evidence (e.g., clinical guidelines); (2) planning to make the practice consistent with the evidence and to change the current practice according to the best evidence, if needed; (3) description of consistent EBP and then dissemination and implementation of the renewed practice during decision-making to improve the consistency between practice and the best evidence; and (4) evaluation and follow up on the practice to establish the best evidence in practice and to ensure that no unjustified variation in practice occurs (Jylhä et al., 2017; Suhonen et al., 2019). The main point is that consistent EBP represents evidence in decision-making (Figure 1). In addition, decisions should consider the patients' experiences and preferences as well as the clinical context (setting), resources, and circumstances. Practitioners (e.g., nursing staff) use all these pieces of information when they, together with their patients, make decisions about their care under multidisciplinary collaboration (Jylhä et al., 2017; Langlois, Daniels \& Akl, 2018).

Ward managers are the bridge between nursing administration and nursing staff in healthcare settings (Engle et al., 2017). They also ensure a context and culture that support the implementation of EBP in nursing at the unit level (Aarons et al., 2014; Duncombe, 2017). In addition, they mediate between strategy and day-to-day activities, and they address concerns raised by staff nurses as well as enable these nurses to fulfil their implementation-related responsibilities (Birken et al., 2018).

Based on the existing reviews (Sandstrom et al., 2011; Birken et al., 2018), one of the important tasks of ward managers is to disseminate information, such as by arranging education for nurses, about the implementation of consistent EBP. Furthermore, they evaluate nurses' response to this information and reinterpret it if necessary (Birken et al., 2018). They also provide support and they communicate the value of EBP in order to facilitate EBP implementation (Innis et al., 2015; Engle et al., 2017; Caramanica \& Spiva, 2018); moreover, they provide resources for EBP (Kueny et.al., 2015; Caramanica \& Spiva, 2018). In addition, ward managers can support nurses by working in alignment with the organization's objective and by acting as an example to nurses by stimulating and guiding them in conducting EBP activities (Stetler et al., 2014). As indicated in the above studies, the ward managers' tasks in leading improvement and in disseminating and implementing EBP consistently are crucial.

However, studies on the tasks of ward managers concerning the use of research (Gifford et al., 2007) and the implementation of EBP (Sandstrom et al., 2011; Birken et al., 2018) at different levels of healthcare organizations remain relatively few. In addition, most studies on EBP have focused on specialized healthcare (Mallion \& Brooke, 2016). Therefore, the present study evaluates the ward manager's tasks in EBP implementation in long -term care facilities, where most nursing staff consist of practical nurses, unlike in acute hospitals. This study aimed to describe the tasks of a ward manager in improving consistent EBP according to the four-phase OMEBP.

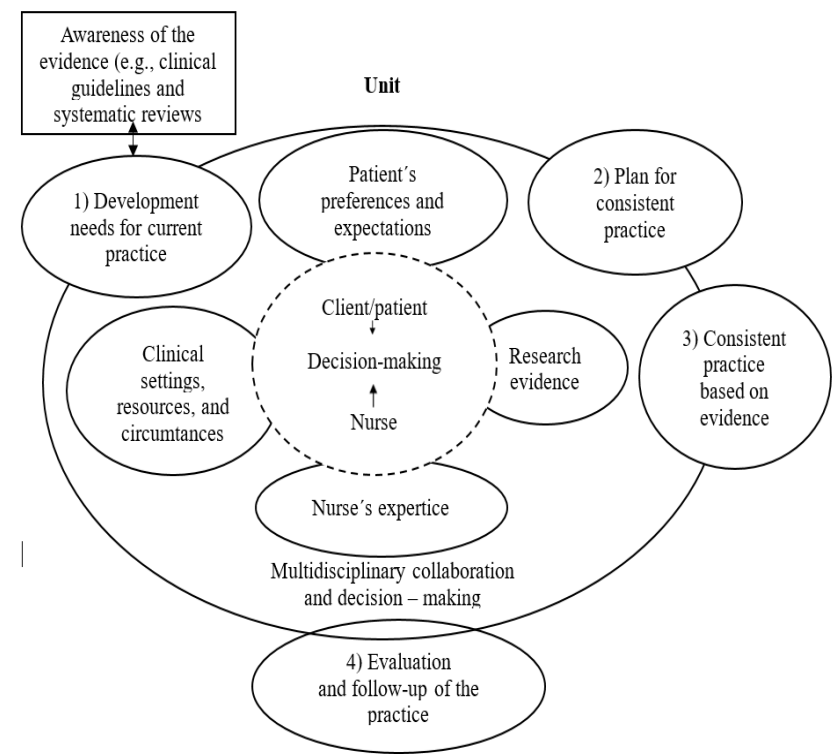

Figure 1: Operational Model for Evidence-Based Practice (OMEBP) (Holopainen et al., 2010; originally published in Finnish). 


\section{METHODOLOGY}

\section{Design}

This study was conducted based on an explorative qualitative design involving focus group interviews.

\section{Data collection}

Data were collected from a long-term care facility for the elderly between February 2016 and January 2017. This care facility consists of 4 units, 126 beds, 4 ward managers, 9 registered nurses, and 67 practical nurses. This study, which included nine focus group interviews, was conducted due to the limited number of studies that have investigated the tasks of ward managers in developing and implementing consistent EBP in longterm care facilities.

Focus group interviews regarding the development and implementation of renewed consistent practice based on international guidelines for pressure ulcer prevention were simultaneously conducted in the four phases of OMEBP (National Pressure Ulcer Advisory Panel, European Pressure Ulcer Advisory Panel, Pan Pacific Pressure Injury Alliance, 2014). Two researchers led the development and implementation of renewed consistent EBP, which lasted 12 months.

The interviews in the four phases were separately conducted in two groups: (1) ward managers $(n=4)$ and (2) wound contact persons [nurses and practical nurses $(\mathrm{n}=8)$ ]; participants from the four units were present in the same interview. The wound contact persons were selected by the ward managers from those who volunteered.

They were organized into a team and, together with the ward managers, they were responsible for the development and implementation of renewed consistent practice. Due to their responsibilities in the development and implementation of consistent EBP, these two groups were interviewed in each of the four phases of OMEBP (a total of eight focus group interviews). The first phase also included a third focus group interview, which involved eight practical nurses. The first phase of the interview was about the development needs in the current practice, and it did not require participation in the actual improvement of consistent practice. Thus, the practical nurses were able to participate in the focus group interview only in the first phase.

Two researchers conducted the focus group interviews. One was the interviewer and the other was an observer, and they were responsible in conducting, documenting, and recording the interviews. Before the interviews began, the participants were informed about the anonymity, confidentiality, voluntary nature, and tape recording of the interviews. They were given a consent form and a brief four-item background questionnaire.

The focus group interviews consisted of the same question, as follows: What are the tasks of a ward manager in the following four phases of OMEBP: (1) determination of the development needs of the current practice to assess whether it is in line with the best evidence (e.g., clinical guidelines); (2) planning to make the practice consistent with the evidence and to change the current practice according to the best evidence, if needed; (3) description of consistent EBP and then dissemination and implementation of the renewed practice during decision-making to improve the consistency between practice and the best evidence; and (4) evaluation and follow up on the practice to establish the best evidence in practice and to ensure that no unjustified variation in practice occurs. All interviews were conducted in a peaceful setting in the nurses' own workplace, and each interview lasted for about 60 minutes.

\section{Data analysis}

Data obtained from all the interviews were combined for analysis. The tasks of ward managers were analyzed by three researchers through deductive content analysis based on the four phases of OMEBP. Within each of the four phases, data were analyzed inductively (Graneheim \& Lundman, 2004) by establishing concepts that described each phenomenon. First, data were read several times to gain a thorough understanding. Second, meaningful units were identified (e.g., words, sentences, and paragraphs), and the data were coded to make it easier to return to the original material. Third, condensed meaningful units were formed, and those with similar content were grouped into categories and named based on their content (Elo \& Kyngäs, 2008).

\section{Ethical considerations}

Ethical approval was sought from the Ethics Committee of Higher Education Institutions (ETMK 126/2015). Permission to collect data was obtained according to the policies of the relevant organizations. The participants were informed about the purpose of this study and they could refuse to participate at any point of the research. Anonymity and confidentiality were ensured in every stage of the research. 


\section{RESULTS}

\section{Characteristics of the participants}

The mean age of the ward managers $(n=4)$ was 53 years, and their mean work experience was 22.8 years. The mean age of the wound contact persons [registered nurses $(n=4)$ and practical nurses $(n=4)$ ] was 42.9 years, and they had been working for an average of 16.7 years. The mean age of the eight practical nurses who participated in the focus group interview in the first phase of the model was 41 years, and their mean work experience was 9.6 years.

Tasks of ward managers in the improvement of consistent practice

The tasks of ward managers are described based on the four-phase OMEBP. These tasks included informing, mentoring, taking responsibility, supporting nursing staff, reminding, and consulting (Figure 2).

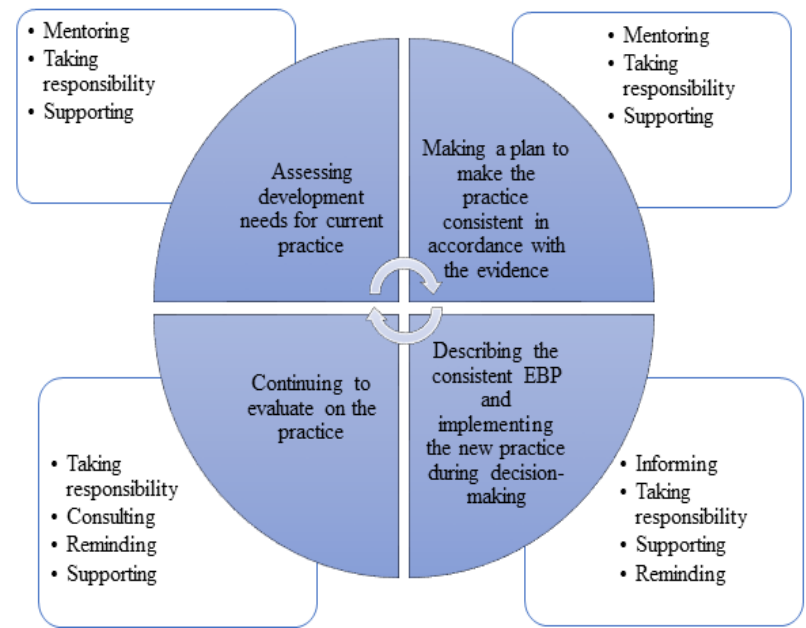

Figure 2: The role of ward nurses in the four-phase Operational Model for Evidence-Based Practices

\section{Development needs of the current practice}

When assessing the development needs of the current practice, the participants identified several tasks for ward managers. These tasks included mentoring, supporting nursing staff, and taking responsibility (Figure 2).

According to the participants, ward managers are expected to mentor nurses about the care lines and provide them general and concrete instructions concerning the nursing practice. They observed that a nurse manager's mentoring task is implemented by participating in formal and informal discussions with nurses regarding the care practice in their own units. One ward manager said:

\section{"Then we try to guide the line of care..."}

The participants agreed that it is the ward manager's task to support nurses by ensuring that nursing practice in the units were updated and based on best evidence. Furthermore, to guarantee the quality of care and the nurses' competence, the ward manager must support the nursing staff in their decision making and in deciding about the care lines. Participants found this as important tasks of the ward manager in order to strengthen and validate the best EBP. As one ward manager said:

"Well, the nurses need to be supported [so] that they can do just how they have been thinking to do and how things have to be done."

Moreover, the participants considered that the ward manager is responsible for the care of residents and all the functions of the unit. In this task, the responsibility of overseeing all nursing practices are included and of taking the initiative to assess the current nursing practice in order to implement the evidence-based guidelines. They stressed that in instances where nursing staff encounter some misunderstanding or other difficulties with the residents or with their relatives regarding the care lines, the ward manager should take over to solve such situations. One registered nurse commented:

“...she then takes responsibility, and she'll start to speak on our behalf..."

\section{Plan for consistent practice}

The tasks identified for a ward manager when planning for consistent practice were the same as in the previous phase: mentoring, supporting nursing staff, and taking responsibility (Figure 2).

According to the participants, the ward manager is expected to mentor the registered and practical nurses by complimenting them when they create a plan to make the practice consistent with the evidence. They agreed that mentoring involves providing pieces of advice and instructions to the nursing staff when they create plan for the renewed consistent practice. One practical nurse said:

"I experience that I can ask advice from [the] ward manager .... that what we should do in this phase and.... it depends on the ward manager..."

Moreover, the participants considered that it is the ward manager's task to provide concrete support to the 
nursing staff by ensuring adequate working hours and by encouraging everyone's participation in the planning process. One ward manager commented:

"it is our duty to arrange time for education so that as many nurses as possible can participate."

The participants agreed that the ward manager should take responsibility of the planning process by selecting the nurses who would participate in the planning; the selected nurses will be trained and will supervise the working group. By responsibility, the participants meant that the ward manager have to observe the planning process and ensure the realization of it. The participants thought that a ward manager, being an observer of the process, must ensure the creation of working groups and group meetings. They also suggested that the ward manager should ensure that the working groups make decisions and that those decisions will be translated into practice. Furthermore, the participants suggested that the ward manager should engage nursing staff in the planning process by bringing important issues into the discussion during unit meetings. They thought that the ward manager should promote the planning by reporting the process to the management. One ward manager said:

"Ward managers have a responsibility to observe what people plan and do, that a working group meets, and that the working group takes care of things."

\section{Implementation of consistent practice based on evidence}

The tasks identified for a ward manager when implementing the renewed consistent practice at the unit included informing, supporting the nursing staff, taking responsibility, and reminding (Figure 2).

The participants found that all residents at the unit and their family members must be informed about the renewed consistent practice. However, they recognized that providing this information and the reason behind the changes is the task of the ward manager.

In addition, the participants thought that a ward manager should orient all new employees about the renewed consistent practice. Equally important is that the ward manager should inform the nurse directors and those who are in the decision-making positions about the renewed practice and its importance. One ward manger commented:

"...informing the personnel and also going to all kinds of meetings, which include more of the people who make decisions."

The participants considered that the development and implementation as well as the updating of the renewed practice require multiple skills from the nursing staff. Therefore, they propose that the ward manager should support and should allow the nursing staff to participate in professional training to update their skills. According to them, an important task for the ward manager is to give the nurses the authority to implement EBP, which also requires practical support from the ward manager. They agreed that it is the ward manager's task to procure the necessary equipment and supplies to realize the operation according to the renewed practice. One practical nurse said:

"... of course, to organize the work shifts so that you can take part in the training."

The participants considered that ward managers should take responsibility to ensure that improved EBP will be implemented to the residents cared for in their units. By this, they meant that a ward manager must orient new nursing staff and offer them pieces of advice if needed. Even though ward managers are not involved in everyday care, the participants expected that the ward managers should facilitate the operation of the unit in the best possible way. They thought that to realize such operation, ward managers should secure enough personnel and plan work shifts so that employees can participate in mutual meetings, where participants would agree on implemented consistent practice. One registered nurse said:

"They'll [ward manager's'] let you [do tasks] that of course you order and do, and they'll give you the possibilities [for the task]."

The participant also suggested that a ward manager should remind nursing staff to follow the renewed consistent practice. They saw this task as important in order to maintain the implementation of the renewed consistent practice in their unit. One practical nurse said:

"And, of course, these things will continue to remind you ... I guess when this is the end of the point ... it won't look like something to forget then ... keep it up."

\section{Evaluation and follow-up of consistent practice}

As regards the evaluation and follow-up of the consistent practice, the following tasks for a ward manager were identified: consulting, taking responsibility, supporting nursing staff, and reminding (Figure 2). 
According to the participants, another task of the ward manager is to provide opportunities for expert consultations and to be available to the nursing staff for such consultations. The participants also commented that the ward manager did not know the residents of the units because of the large number of residents and their failure to participate in daily nursing practice. Therefore, they suggested that the ward manager should be available for consultations. This means that ward managers are expected to share their expertise with the staff and regularly participate in the evaluation of resident care. According to the participants, the ward manager, together with their colleagues and nurse directors, should evaluate the renewed consistent practice. One registered nurse said:

\section{"Quite a bit.... with each other we are going through things here."}

The participants suggested that ward managers must take primary responsibility for nursing interventions in the unit. In this task, taking responsibility is evident in keeping care lines that were agreed upon and in informing the staff, the residents, and their relatives as well as the members of the decision-making body about the current renewed consistent practice. One ward manager commented:

"It's a little like the responsibility for nursing lies with us..."

Moreover, the participants expected that the ward manager should support the nursing staff in evaluating the renewed consistent practice. By this, they meant that the ward manager should make decisions regarding resident care and the nurses' role in the resident care chain. They emphasized that the ward manager should provide positive feedback to the nursing staff given that feedback related to success indicates that the staff were in the right direction in terms of practice, and it motivates them to continue to observe the renewed consistent practice. One ward manager said:

"...the encouragement of nursing staff all the time so that they'll remember their own task in the care chain..."

The participants expected that the ward manager will remind the staff of the planned and agreed upon practice, of the utilization of the multiprofessional team, and of the recognition of the implemented practice in patient documentation. Their opinion was that the ward manager should remind the staff and clarify problematic issues, monitor practice, and delegate responsibility to the nursing staff during unit meetings and other joint discussion sessions. One ward manager said:

"[We always need to] remind and remind people that something needs to be done..."

\section{DISCUSSION}

This study aimed to describe the tasks of ward managers in improving consistent EBP according to the four-phase OMEBP. The results of this study supported those of several previous studies (e.g., Duncombe, 2017; Aarons et al., 2014) that highlighted the ward managers' responsibility in EBP implementation. The present results showed that taking responsibility in every phase of the process at the ward level is an important task of the ward manager.

The results indicated that one of the ward manager's task is to take responsibility to take care of residents and thus for the nursing recommendations and for improving and implementing consistent EBP. These results are supported by a previous review concerning the middle manager's role in implementing EBP in healthcare (Birken et.al., 2018)

Providing consultation and expert support to nursing staff to facilitate the implementation of EBP has been reported to be an important task of ward managers (Engle et al., 2017; Caramanica \& Spiva, 2018). In the present study, this task was also expected of ward managers in every phase of the improvement process. Moreover, the results indicated that provision of concrete resources, such as time, equipment, and supplies, to facilitate work in accordance with the renewed consistent practice was a task of the ward manager, consistent with previous findings (Kueny et. al., 2015; Caramanica \& Spiva, 2018).

In this study, the ward manager's responsibilities included mentoring nurses in all phases of the improvement process, consistent with previous findings (Sandstrom et al., 2011; Birken et al., 2018). Studies have shown that by acting as role model and by valuing research, a ward manager can encourage utilization of research among nurses (Gifford et al., 2007). Similar results were found in the present study wherein mentoring was deemed a task of the ward manager, and this task included participation in formal and informal discussions concerning nursing practice in their units. A ward manager could also mentor by motivating nurses, by showing the care lines, and by providing instructions.

Reviews (Sandstrom et al., 2011; Birken et al., 2018) have shown that ward managers must overcome obstacles, hold staff accountable, and coach staff nurses. 
In the present study, provision of positive feedback as acknowledgement of good practice of the staff was an important task of the ward manager. Moreover, according to Engle et al., (2017) nurses can be encouraged to learn and use improvement tools through training and coaching, which were also seen as important tasks of the ward manager in the present study.

A previous review (Birken et al., 2018.) has found that informing about EBP is a task of the ward manager that is, converting information into a more general form and reinterpreting it if necessary, through feedback. Similarly, informing the new employees, the management, and the decision makers about the renewed consistent practice was also a task of the ward manager in this study. However, compared with previous studies, informing the residents and their relatives about the renewed consistent practice were found to be a new task of the ward manager.

The present results also showed that reminding the staff to comply with the consistent practice and with the multidisciplinary collaboration during the entire improvement process is the ward manager's task. Moreover, the manager must clarify issues, to monitor practice, and to delegate responsibility during ward meetings and other joint discussion sessions. In a review, Sandstrom et al., (2011) reported similar tasks for a ward manager, namely, being present and visible and being able to communicate adequately to facilitate EBP implementation.

Studies have shown that ward managers play a key role as the bridge between the nursing administration and the nursing staff (Engle et al., 2017). The present results showed that ward manager must act as experts, which mean that they had to share their expertise with the nursing staff and had to discuss with the nurse administrators. Another task of the ward manager was to support the nurses in cases of misunderstandings or other difficulties with the residents or their relatives regarding the care lines or other issues.

Ward managers play a crucial role in implementing EBPs in long-term care facilities, which is why they are presumed to tap their extensive knowledge on EBPs to integrate nursing practice and procedures. An interesting result of this study is that a ward manager was expected to provide support to nursing staff in all phases. The present study showed that there is a need for supporting mechanisms (e.g., mentoring and training programs) and processes (e.g., follow-up and evaluation systems) to implement the best practices in daily operation, as presented in previous reviews (Sandstrom et al., 2011; Birken etal., 2018).

On the broader policy and social and health care service levels, the present results are important in order to promote EBP and quality of care. Studies have shown that although nurses intend to implement EBP, they need to be appreciated for their work, and the working culture has to support it (Morgan, 2012). A ward manager is in the crucial position to improve the motivation of nurses to provide the best possible care for their clients. This study showed that the tasks identified for a ward manager were consistent with those reported in previous studies. However, the tasks should be described systematically and in greater detail so that the knowledge can be used in education and training for ward managers. The tasks of a ward manager should also be clear and well known among all staff.

\section{Limitations}

Focus group interviews were conducted after each phase of the improvement process of renewed consistent practice according to the OMEBP model. As the improvement process was conducted in only one organization, the number of informants was small. The entire process lasted 12 months. An interviewer and an observer conducted all of the interviews; they complemented each other's functions, and they facilitated a clear discussion. Data were analyzed using deductive and inductive content analyses. Three researchers performed the analyses (Elo et al., 2014). Dependability refers to the consistency of the research process and method. Thus, the interview questions were the same in all phases. Dependability was verified through an exact description of the research project. Context description allowed assessment of the transferability of the results (Graneheim \& Lundman, 2004).

\section{CONCLUSION}

This study contributes to the limited literature on the tasks expected of ward managers in the implementation of consistent EBPs in long-term care facilities. According to the findings, ward managers play a crucial and versatile role in each of the four phases of OMEBP in improving consistent EBPs. Such tasks as assessing the current practice, improving the renewed consistent practice, and implementing and evaluating the new practice demand different kinds of competence from ward managers. 


\section{REFERENCES}

Aarons, G.A., Farahnak, L.R., Ehrhart, M.G. \& Sklar, M. (2014). Aligning leadership across systems and organizations to develop strategic climate for evidence-based practice implementation. Annual Review of Public Health, 35, pp $255-274$

Andermann, A., Pang, T., Newton, J.N., Davis, A. \& Panisset, U. (2016). Evidence for health II: Overcoming barriers to using evidence in policy and practice. Health Research Policy and Systems, 14, 17.

Birken, S., Clary, A., Tabriz, A.A., Turner, K., Meza, R., Zizzi, A., Larson, N., Walker, J. \& Charns, M. (2018). Middle manager's' role in implementing evidence-based practice in healthcare: a systematic review. Implementation Science, 13(1), 149.

Burns, N. \& Grove, S. (2013). Burns and Grove's understanding nursing research: Building an evidence-based practice. Philippine Edition by Teresita I. Barcelo. Singapore: Elsevier Inc.

Caramanica, L. \& Spiva, L. (2018). Exploring nurse manager support of evidence-based practice: Clinical nurse perceptions. Journal of Nursing Administration, 48(5), pp 272-278.

Duncombe, D.C. (2017). A multi-institutional study of the perceived barriers and facilitators to implementing evidence-based practice. Journal of Clinical Nursing, 27(5-6), pp 1216-1226.

Elo, S. \& Kyngäs, H. (2008). The qualitative content analysis process. Journal of Advanced Nursing, 62(1), pp $107-115$.

Elo, S., Kääriänen, M., Kanste, O., Pölkki, T., Utrianien, K. \& Kyngäs, H. (2014). Qualitative content analysis: A focus on trustworthiness. SAGE Open, 4(1), pp 1-10.

Engle, R.L., Lopez, E.R., Gormley, K.E., Chan, J.A., Charns, M.P. \& Lukas, C.V. (2017). What roles do middle manager's play in implementation of innovative practice? Health Care Management Review, 42(1), pp 14-27.

Gifford, W., Davies, B., Edwards, N., Griffin, P. \& Lybanon, V. (2007). Managerial leadership for nurses' use of research evidence: An integrative review of the literature. Worldviews on Evidence-Based Nursing, 4(3), pp $126-145$.

Graneheim, U. H. \& Lundman, B. (2004). Qualitative content analysis in nursing research: Concepts, procedures and measures to achieve trustworthiness. Nurse Education Today, 24(2), pp 105-112.

Holopainen, A., Korhonen, T., Miettinen, M., Pelkonen, M. \& Perälä, M-L. (2010). Hoitotyön käytännöt yhtenäisiksi toimintamalli näyttöön perustuvien käytäntöjen kehittämiseksi. Premissi 1, pp 38-45.

Heiwe, S., Kajermo, K.N., Tyni-Lenné, R., Guidetti, S., Samuelsson, M., Andersson, I-L. \& Wengström, Y. (2011). Evidence-based practice: Attitudes, knowledge and behaviour among allied health care professionals. International Journal for Quality of Health Care, 23(2), pp 198-209.

Innis, J., Dryden-Palmer, K., Perreira, T. \& Berta, W. (2015). How do health care organizations take on best practice? A scoping literature review. International Journal of Evidence-Based Healthcare, 13(4), pp 254-272.

Institute of Medicine (US) Roundtable on Evidence-Based Medicine (2009). Leadership Commitments to Improve Value in Healthcare: Finding Common Ground: Workshop Summary. Washington, DC: National Academies Press (US). Retrieved from: https://www.ncbi.nlm.nih.gov/books/NBK52847/

Jun, J., Kovner, C.T. \& Stimpfel, A.W. (2016). Barriers and facilitators of nurses' use of clinical practice guidelines: An integrative review. International Journal of Nursing Studies, 60, pp 54-68.

Jylhä, V., Oikarainen, A., Perälä, M-L. \& Holopainen, A. (2017). Facilitating evidence-based practice in nursing and midwifery in the WHO European Region. Denmark: WHO Regional Office for Europe. Retrieved from: http://www.euro.who.int/_data/assets/pdf_file/0017/348020/WH06_EBP_report_complete.pdf?ua=1

Kalisch, B.J., Tschannen, D., Lee, H. \& Friese, C.R. (2011). Hospital variation in missed nursing care. American 
Journal of Medical Quality, 26(4), pp 291-299.

Kueny, A., Shever, L.L., Mackin, M. \& Titler, M. (2015). Facilitating the implementation of evidence-based practice through contextual support and nursing leadership. Journal of Healthcare Leadership, 24(7), pp 29-39.

Langlois, E.V., Daniels, K. \& Akl, E.A. (2018). Evidence synthesis for health policy and systems: A methods guide. Geneva: World Health Organization. Retrieved from: https://www.who.int/alliance-hpsr/resources/publications/ Alliance-evidence-synthesis-MethodsGuide.pdf

Mallion, J. \& Brooke, J. (2016). Community- and hospital-based nurses` implementation of evidence-based practice: are there any differences? British Journal of Community Nursing, 21(3), pp 148-154.

Morgan, L.A. (2012). A mentoring model for evidence-based practice in a community hospital. Journal for Nurses in Staff Development, 28(5), pp 233-237.

National Pressure Ulcer Advisory Panel, European Pressure Ulcer Advisory Panel, Pan Pacific Pressure Injury Alliance. (2014). Prevention and treatment of pressure ulcers: Quick reference guide, $2^{\text {nd }}$ edition. Haesler, E. (Ed.). Perth, Australia: Cambridge Media.

Sandstrom, B., Borglin, G., Nilsson, R. \& Willman, A. (2011). Promoting the implementation of evidence-based practice: A literature review focusing on the role of nursing leadership. Worldviews on Evidence-Based Nursing, 8(4), pp 212-223.

Stetler, C.B., Ritchie, J.A., Rycroft-Malonen, J. \& Charns, M.P. (2014). Leadership for evidence-based practice: Strategic and functional behaviors for institutionalizing EBP. Worldviews on Evidence-Based Nursing, 11(4), pp 219-226.

Stevens, K.R. (2013). The impact of evidence-based practice in nursing and the next big ideas. Online Journal of Issues in Nursing, 18(2), pp 4.

Suhonen, R., Ylönen, M., Jalonen, L. \& Holopainen, A. (2019) Leading evidence-based practice in Finnish healthcare. In Hafsteinsdóttir H, Jónsdóttir H, Kirkevold M, Leino-Kilpi H, Lomborg K, Rahm Hallberg I (Eds.), Leadership in Nursing: Experiences from the European Nordic Countries, pp 83-98.

Supplee, L.H. \& Metz, A. (2015). Opportunities and challenges in evidence-based social policy. Social Policy Report, 28(4), pp 1-16.

Van Achterberg, T., Schoonhoven, L. \& Grol, R. (2008). Nursing implementation science: How evidence-based nursing requires evidence-based implementation. Journal of Nursing Scholarship, 40(4), pp 302-310.

Veermah, V. (2016). The use of evidence-based information by nurses and midwives to inform practice. Journal of Clinical Nursing, 25(3-4), pp 340-350.

Wang, M.Y., Kao, C.C. \& Lin, C.F. (2015). The EPCOR model: A model for promoting the successful implementation of evidence-based nursing in hospital-based settings. Journal of Nursing Research, 23(1), pp 15-24. 